\title{
Motion processing picks up speed in the brain
}

Recordings of individual neurons in the mouse brain reveal a main mechanism for motion processing in the primary visual cortex. These findings are likely to have implications for other species. SEE ARTICLE P.80

\section{JOSE MANUEL ALONSO}

$\mathrm{I}$ magine life without visual motion. You can easily recognize a face, but you cannot safely cross the street because you do not see cars moving. Instead, you see snapshots of stationary cars that change position at unpredictable times. Most of us do not have this problem, because healthy brains contain many neurons that are direction selective - they fire strongly when they detect movement in one direction (known as their preferred direction, towards the left of the eye's field of view, for instance), and relatively weakly in response to movement in the opposite direction ${ }^{1}$. These directionselective neurons are found in different parts of the brain, including the cerebral cortex. On page 80 , Lien and Scanziani ${ }^{2}$ report a major advance in our understanding of how neurons in the brain's primary visual cortex become direction selective.

Sensory information is transmitted from the eye to the primary visual cortex through a neuronal structure called the thalamus. Thalamic and cortical neurons respond only to stimuli within a small portion of the field of view, known as their receptive field. Previous stud$\mathrm{ies}^{3-7}$ in cats suggest that direction selectivity in a cortical neuron arises from the combined activity of multiple thalamic neurons, which converge on the cortical neuron. These thalamic inputs have overlapping receptive fields, do not have direction selectivity themselves, and are temporally diverse in terms of their responses to stimuli. In this model, different thalamic inputs responding to moving stimuli over differing time periods generate a preferred direction of movement in the cortical neuron. However, finding direct experimental evidence for this model has been technically challenging.

Lien and Scanziani have overcome the technical limitations of the past thanks to the emergence of powerful tools that enable researchers to genetically manipulate neurons and measure the activity of multiple thalamic inputs to one cortical neuron. First, the authors used genetic-engineering techniques to silence the non-thalamic inputs to the primary visual cortex of mice, so that they could isolate the effects of thalamic inputs on direction selectivity in cortical neurons. These experiments replicated previous findings in cats ${ }^{4}$, confirming that cortical cells maintain their direction selectivity when non-thalamic inputs are inactivated. Thus, direction selectivity in the primary visual cortex of mice originates at thalamic inputs.

Next, the researchers investigated the neuronal mechanisms underlying cortical direction selectivity in mice. In cats and primates $^{8-10}$, some regions of the cortical receptive field respond more slowly and less transiently to visual stimuli than do other regions. The cortical neurons are preferentially activated by stimuli moving from slow- to fast-responding regions, because these stimuli produce the maximum neuronal response through temporal synchronization. To understand this synchronization, imagine two people who want to clap at the same time but who, when called to clap, respond at different rates - one more slowly than the other. To synchronize the
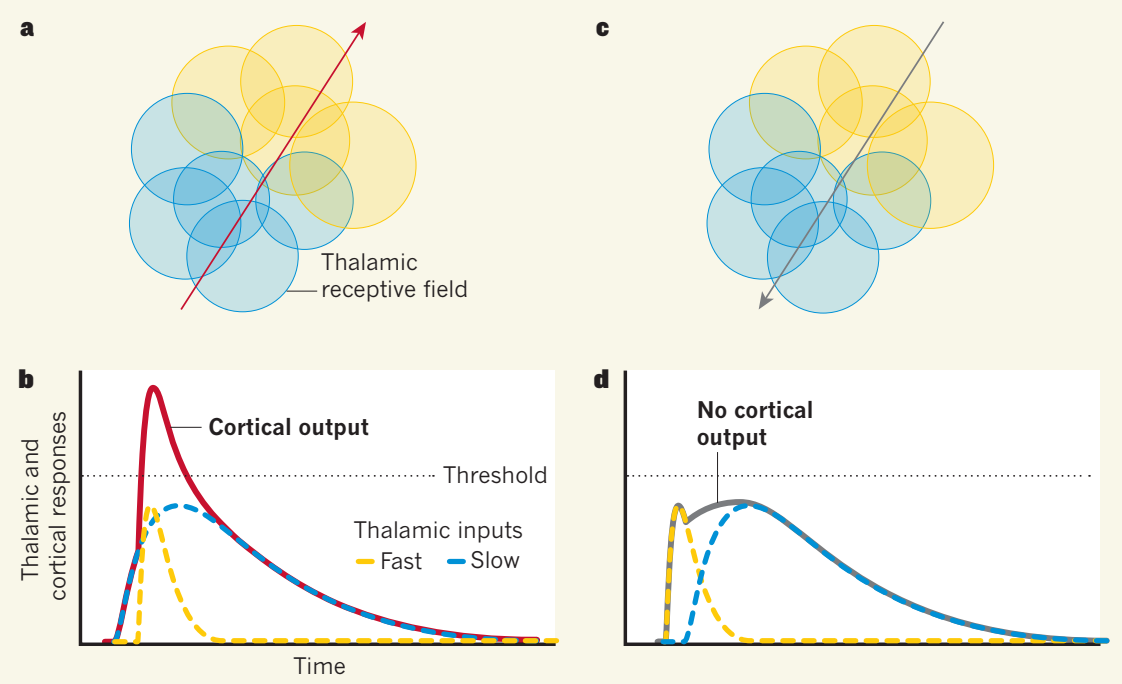
response decay.

c

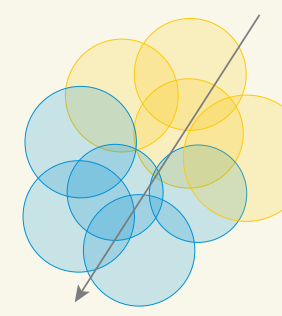

claps for maximum impact, the slower clapper has to receive the signal first, and the faster clapper, second. As in cats and primates, Lien and Scanziani found that cortical neurons in mice prefer a moving direction that goes from slow to fast regions of the receptive field.

When the authors silenced non-thalamic inputs, each cortical neuron still had slow and fast regions within its receptive field and preferred moving stimuli that crossed the slow region first. This finding shows, for the first time, that thalamic inputs are sufficient to generate both slow and fast responses in different regions in the cortical receptive field, and that these regions generate direction selectivity through synchronization. The authors also demonstrated that the temporal differences between the responses of different cortical receptive-field regions were most pronounced at the end of a response to a stimulus (the response decay): activity generated by slow regions decayed more gradually than activity generated by fast regions. These findings are consistent with work in cats and primates ${ }^{7,9}$ that suggests that cortical direction selectivity is reliant mainly on synchronization of the

In their final and most impressive experiments, Lien and Scanziani recorded simultaneously the activity of a single cortical neuron and its thalamic inputs. The beautiful data collected in these experiments provide the strongest evidence so far that the slow and fast regions of the cortical receptive field

Figure 1 | Generating direction selectivity in the brain's primary visual cortex. Neurons in the primary visual cortex respond to movements in a particular direction within a small portion of the eye's field of view - their receptive field. Lien and Scanziani ${ }^{2}$ have revealed a main mechanism for direction selectivity in this brain region in mice. a, Neurons in the brain's thalamus that have overlapping receptive fields send inputs to a single cortical neuron. Their receptive fields can respond to movement in either a fast, transient manner (yellow) or a slow, sustained manner (blue). The authors demonstrate that cortical neurons are preferentially activated by movement in the direction (red arrow) that causes slow thalamic inputs to respond first, followed by fast inputs. b, As the graph shows, this is because signals from both types of input are temporally synchronized, maximizing the cumulative cortical response (red line), which crosses the threshold required to generate an electrical output. c, By contrast, movement in the opposite direction (grey arrow) activates the fast thalamic inputs first. $\mathbf{d}$, This generates a weaker cortical response (grey line), which does not cross the threshold because the thalamic inputs are less synchronized, and so does not produce an electrical output. 
are generated by multiple thalamic inputs that have temporally different responses to the stimulus (Fig. 1). Thalamic inputs that respond slowly to visual stimuli generate slow responses in cortical regions, whereas those responding faster generate fast responses.

Lien and Scanziani's results, taken together with previous work ${ }^{3-10}$, raise the interesting possibility that cortical direction selectivity is generated through a common mechanism - the convergence of temporally diverse thalamic inputs - in rodents, cats and primates. But as with all research, some questions remain open.

For instance, the authors focus their study on the middle layers of the visual cortex, which receive the bulk of the thalamic input ${ }^{11}$. As Lien and Scanziani show, many thalamic inputs in these middle cortical layers are not direction selective, but their combined activity is. It remains unclear whether thalamic inputs that target other cortical layers (or serve other functions) can encode direction selectivity through different mechanisms. For example, neurons in the superficial layers of the cortex might derive their direction selectivity from thalamic neurons that are themselves direction selective ${ }^{12}$.

It is also known that thalamic inputs to the visual cortex are arranged by their receptivefield position - inputs that have receptive fields close to one another in the field of view are clustered together. However, it is not yet known whether the thalamic inputs are also arranged according to their temporal properties. If so, this could explain why spatial position and direction preference tend to change together in different neurons across the visual-cortical map ${ }^{13}$.

Whatever the answers are, it is becoming increasingly clear that the visual cortex generates stimulus selectivity, such as preferences for direction and orientation, through thalamo-cortical convergence. Lien and Scanziani's work shows that this mechanism is better preserved across mammals than was previously thought.

Jose Manuel Alonso is in the College of Optometry, State University of New York, New York, New York 10036, USA.

e-mail:jalonso@sunyopt.edu

1. Hubel, D. H. \& Wiesel, T. N. J. Physiol. 160, 106-154 (1962).

2. Lien, A. D. \& Scanziani, M. Nature $\mathbf{5 5 8 , 8 0 - 8 6}$ (2018).

3. Alonso, J.-M., Usrey, W. M. \& Reid, R. C. J. Neurosci. 21, 4002-4015 (2001).

4. Ferster, D., Chung, S. \& Wheat, H. Nature $\mathbf{3 8 0}$, 249-252 (1996).

5. Saul, A. B. \& Humphrey, A. L. J. Neurophysiol. 64. 206-224 (1990)

6. Saul, A. B. \& Humphrey, A. L. J. Neurophysiol. 68 1190-1208 (1992)

7. Stanley, G. B. et al. J. Neurosci. 32, 9073-9088 (2012).

8. Reid, R. C., Soodak, R. E. \& Shapley, R. M. J. Neurophysiol. 66, 505-529 (1991).

9. Livingstone, M. S. Neuron 20, 509-526 (1998).

10.McLean, J. \& Palmer, L. A. Vision Res. 29, 675-679 (1989).

11.Lorente de No, R. In Physiology of the Nervous System (ed. Fulton, J.) 291-340 (Oxford Univ. Press, 1938).

12.Cruz-Martín, A. et al. Nature 507, 358-361 (2014).

13. Kremkow, J., Jin, J., Wang, Y. \& Alonso, J. M. Nature 533, 52-57 (2016).

This article was published online on 23 May 2018.

\section{Two artificial synapses are better than one}

\section{Emerging nanoelectronic devices could revolutionize artificial neural networks, but their hardware implementations lag behind those of their software counterparts. An approach has been developed that tips the scales in their favour. SEE ARTICLE P.60}

\section{GINA C. ADAM}

I nspired by the brain's neural networks, scientists have for decades tried to construct electronic circuits that can process large amounts of data. However, it has been difficult to achieve energy-efficient implementations of artificial neurons and synapses (connections between neurons). On page 60, Ambrogio et al. ${ }^{1}$ report an artificial neural network containing more than 200,000 synapses that can classify complex collections of images. The authors' work demonstrates that hardware-based neural networks that use emerging nanoelectronic devices can perform as well as can software-based networks running on ordinary computers, while consuming much less power.

Artificial neural networks are not programmed in the same way as conventional computers. Just as humans learn from experience, these networks acquire their functions from data obtained during a training process. Image classification, which involves learning and memory, requires thousands of artificial synapses. The states (electrical properties) of these synapses need to be programmed quickly and then retained for future network operation.

Nanoscale synaptic devices that have programmable electrical resistance, such

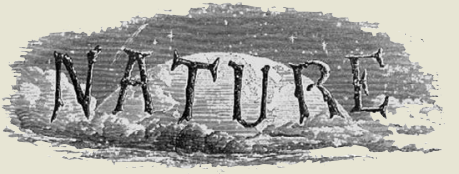

50 Years Ago

Reading aids for the blind have so far involved the use of intact sensory pathways and have progressed little beyond Braille and tape-recorded "talking-books". Both these systems are quite expensive ... and both are slow in terms of information transfer to the reader ... At a recent meeting of the Physiological Society, Brindley and Lewin demonstrated a device for stimulating the visual cortex of man directly ... Essentially it consists of an array of radio receivers, encapsulated in silicone rubber and screwed to the skull ... Activation of a receiver stimulated the cortex: transmission was in the form of a train of short $(200 \mu \mathrm{s})$ pulses ... it does at least seem feasible to transmit visual information directly to the central visual pathways of the recently blind.

From Nature 8 June 1968

100 Years Ago

It happened last week that about $1 \mathrm{lb}$. of fresh lamb was put into an oven at night in order that it might be cooked by morning on the "hay-box" principle. It was in a casserole, with a little water. Similar treatment in the same oven on previous occasions had been very successful. At about 5 a.m. the casserole was examined, and the broth was found to be very well tasted, and the whole smelt fresh and good, but the meat when tested with a fork was not tender, and the fat (of which there was a good deal) was entirely unmelted. The casserole was returned to the oven (then quite cool) and taken out again after breakfast. The contents were then found to be smelling most offensively, as if extremely "high". The fat was melted. The meat and broth were judged quite unfit for human food. I wonder if any of your readers would explain this curious development.

From Nature 6 June 1918 